\title{
The Development of Experimental Economics-Based on the Econometric Analysis of SCl and SSCI Database
}

\author{
Si-Yang SONG ${ }^{1, a}$, Ling-Xue SUN ${ }^{2, b, *}$ \\ ${ }^{12}$ School of Public Affairs, Zhejiang University, Yuhangtang Road 866, 310058 Hangzhou, China \\ ${ }^{2}$ School of Public Affairs, Zhejiang University, Yuhangtang Road 866, 310058 Hangzhou, China \\ asongsy@zju.edu.cn, bsunlx@zju.edu.cn \\ *Ling-Xue SUN
}

Keywords: Experimental economics, Social network analysis, Co-occurrence analysis.

\begin{abstract}
Based on the 1328 papers with keywords of "experimental economics", we analyze the 6134 valid keywords by means of bibliometric, and get the high frequency word cloud distribution and co-word network of experimental economics. At present, auction, altruism, risk, reciprocity and so on are the main branches of experimental economics. And the tendency to subdivide of the study theme promotes the traditional theme of the common frequency reduction and the new high-frequency theme emergence. Researchers can rely on the position of a keyword in the network to focus on those keywords that are directly related to it to ensure that the scope of the study covers the existing core issues as much as possible. From the perspective of disciplinary development, experimental economics has become a recognized paradigm of economic research, but its scope of application and internal differentiation is still limited.
\end{abstract}

\section{Introduction}

The development of the discipline is a gradual process. Almost all disciplines have gone through four stages: the ideological revolution, the carrier record, the spread of extension, and recognition [1], so experimental economics is also like this. The acceptance of economics for experimental methods is not natural. The metrological method, which has begun to emerge from the Great Depression in the 1930s, has so far been the mainstream paradigm of economic analysis. It is based on the real data for analysis, which accords with the observation - abstract analysis that people are familiar with. Due to the existence of self-reinforcing mechanisms, the starting metrological paradigm is widely used and locked [2], while experimental economics is suppressed as a new idea of different assumptions [3]. It was not until the 1960s that experimental economics began to emerge, mainly because of the competing relationship between economic principles, and that researchers needed a way to choose the right theory $[4,5]$.

The development of experimental economics relies on the promotion of two disciplines: game theory and psychology. On one hand, game theory is an analysis of rational behavior in different situations, including the analysis of interdependent results, and then its simple and clear theoretical predictions can be easily tested in controlled laboratories $[6,7,8]$. On the other hand, the introduction of the empirical method of psychology translates this possibility into reality, where the introduction of experiment and the monetary payment that is consistent with the behavior is introduced into the experimental economics approach. The experimental method of economics also has its own uniqueness, and the most obvious one is the introduction of value that makes people behave as they are in the maximized expected utility $[9,10]$.

With the possibility and foundation, the embryonic development of experimental economics began. The early representative of the event is Chamberlain's classroom experiment as well as the Santa Monica meeting, and the former is the first experiment of economics while the latter includes a few scholars who have significant impact on discipline development [4,11]. During this period, experimental economics has been relatively slowly through the first two stages of the development of the discipline. By the 1980s, the situation has changed significantly, from the number of published paper increased rapidly. From the external conditions, the use of computer and Internet technology 
expand the analysis of variables, which makes the complex research possible. Within the disciplines also established three research base with the leading of Vernon Smith, Charles Plott and Relnhard Selten, and then cultivate a large number of researchers engaged in experimental economics. Since that the experimental economics has entered the fourth stage.

\section{Research Method}

\section{Co-occurrence Analysis Method}

Co-occurrence analysis is a kind of literature information measurement method, which is based on the analysis of a group of scholars and their articles, and then gets the internal structure of the study discipline. Co-occurrence analysis includes co-citation, co-word and co-link matrices. This paper mainly deals with the second approach.

Co-word analysis refers to a method of analyzing common phrases or words clustering, where high-frequency phrases represent central issues in this field. As a representative method of co-occurrence analysis, co-word analysis was first applied in the field of bibliometrics, and Lesk studied the application of word co-occurrence in information retrieval as early as 1969 [12]. With the increasing of article normalization, on the one hand, the author will list some phrases reflecting the core idea or research method at the beginning of the article. On the other hand, the development of computer technology promotes the automatic searching and adding of the paper keywords. These two ways that provide the keyword information will be reflected in the literature database, which helps us grasp the theme of the article more accurately.

Based on the co-word analysis method, if the keywords $i$ and $j$ are the keywords from the same article, then it is considered that there is a co-word relationship between $\mathrm{i}$ and $\mathrm{j}$. After summarizing all the target documents in the same situation, we will get the co-citation frequency of $i$ and $j$. The specific formula is as follows:

$$
\operatorname{cowo}_{\mathrm{ij}}=\sum_{\mathrm{p}=1}^{\mathrm{n}} \min (\mathrm{Pi}, \mathrm{Pj})
$$

Eq.1, Cowoij refers to the number of times the keyword $\mathrm{i}$ and the keyword $\mathrm{j}$ are cited, where $\mathrm{Pi}$ is the number of occurrences of the keyword $\mathrm{i}$ in the all $\mathrm{P}$ paper keywords, and $\mathrm{Pj}$ refers to the number of occurrences of the keyword $\mathrm{j}$ in the all $\mathrm{P}$ paper keywords, and $\mathrm{n}$ is the total number of articles.

\section{Social Network Analysis Method}

Social network analysis is mainly used to analyze the relationship between one or more subjects and their relationships as well as the patterns and meanings of these connections [13]. This approach in social science helps to explain the relationship between actors and the flow of resources and elements through actors. The traditional quantitative analysis emphasizes some kind of attribute between independent individuals, which separates the interrelationships between things and leads to low socialization. While traditional qualitative research is carried out by analyzing the conceptual data of the actors and it is easy to exaggerate the actors' subjective initiative which leads to excessive socialization. In order to avoid these two problems, the best way to study social action is social network analysis [14].

\section{The Experimental Economic Family: Members and Relations}

The focus of a discipline is closely related to the keywords contained in the paper, and the key words reflect the areas, methods and main contents of the article. We extract 6133 keywords except the "experimental economics" from all the articles, which include 2990 unique ones. After analyzing these keywords preliminarily, it is found that there are two main problems. First, there is the whole meaning group expressing the same core word, and the majority of the words in the meaning group express the same meaning. Second, the occurrence frequency of more than $75 \%$ of the keywords is 1 , and they are located in the fat tail as the "scattered in most of the words". For the former one, we will merge the same meaning parts of the word to strengthen the core meaning; for the latter one, we will set the threshold before the analysis, and then screen the high-frequency part. 
The distribution of Figure 1 shows that, behavior, auction, decision making, game theory, and cooperation are the first five words to be captured. From the point of view of "behavior", the analysis object of experimental economics is individual's decision-making behavior, and any change in the experiment by the observer will be regarded as the choice of behavior under the established conditions. The process also includes the "rational" or "boundless rational" assumptions, the external environment factor "field experiment" or "laboratory experiment", "cooperation" or "betray" strategy in "decision making" part, and "Willingness to pay" and "preference" in the decision-making effect. "Auction" is the first kind of behavior to be used in the experiment. Moreover, the auction of experimental simulation is also most likely to be used in real events. Besides that, it also includes public goods supply, consumer behavior, and investment and so on. The emergence of "game theory" is a good proof of its theoretical support for the development of experimental economics. Experiments with good design can simultaneously explain the behavior and reproduce the reality of the theory.

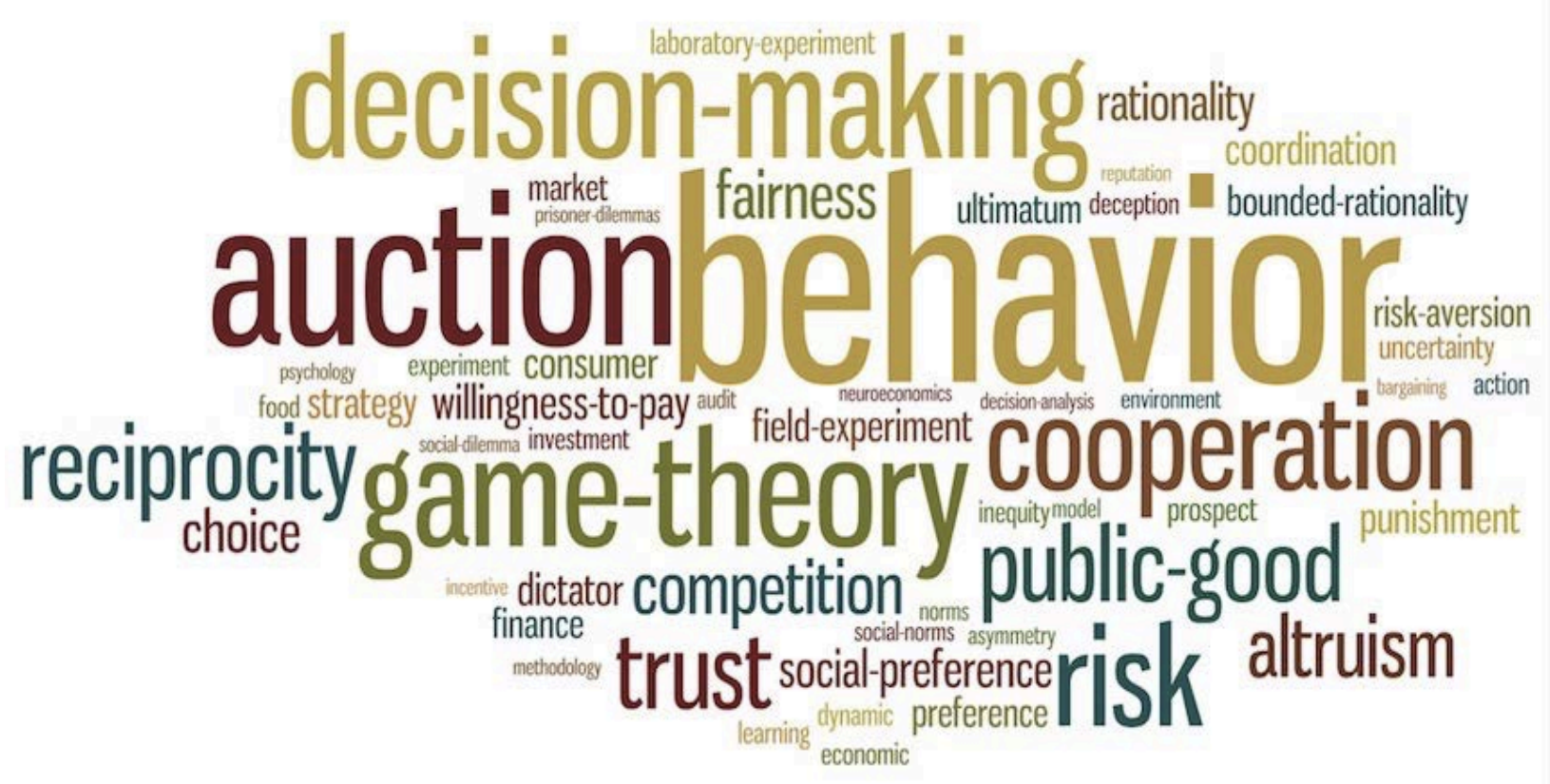

Fig.1 Word cloud of bigrams that contain experimental economics” in paper keywords. Data were derived from the Web of Science on 25-3-2017. 


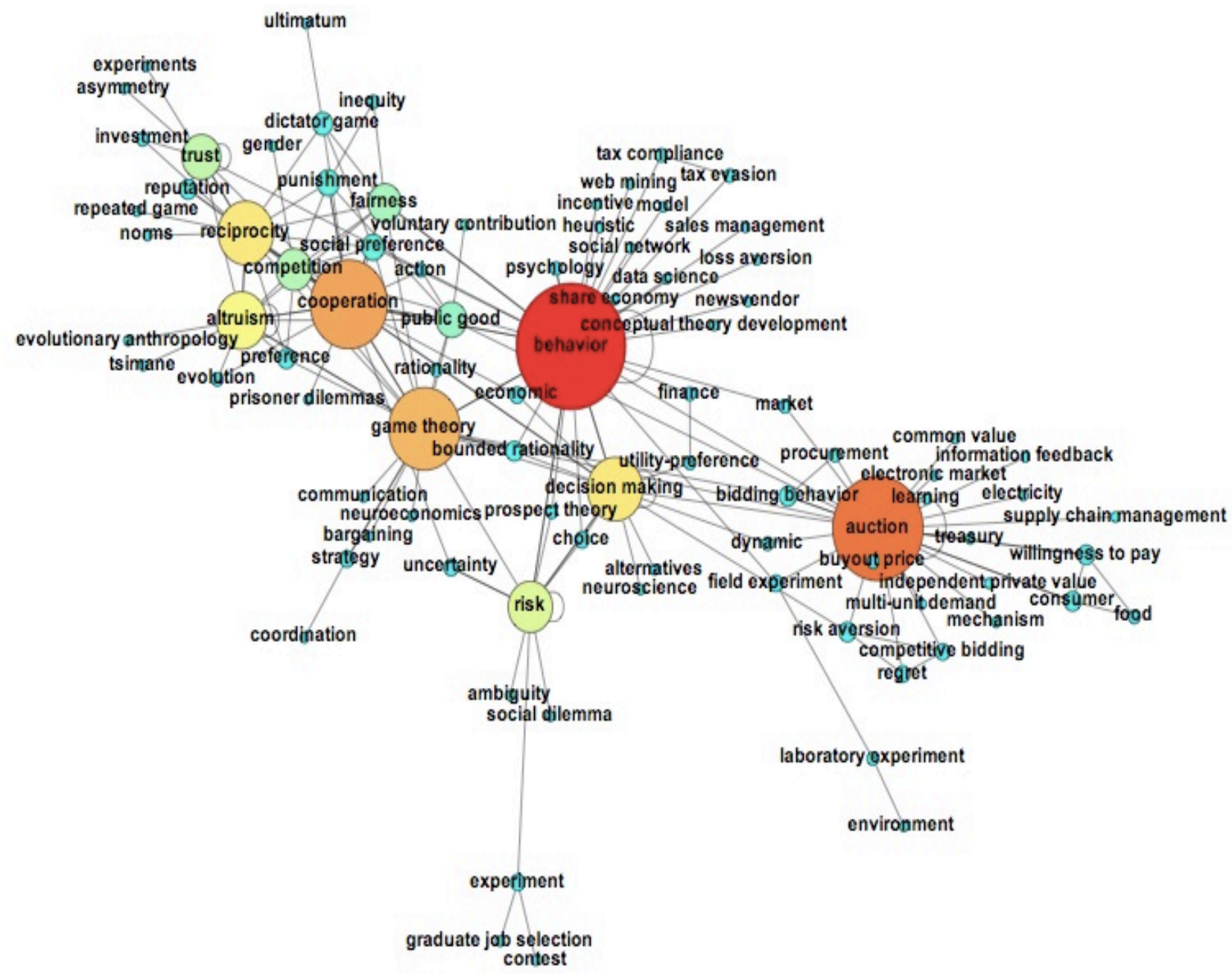

Fig. 2 The co-word map of experimental economic study shows above. We analyze 1,328 selected papers, which are linked by 28,912 keywords with 109 nods and 200 directed and weighted links. Nodes are connected with the theme in each of the clusters and between clusters. Data are obtained from the ISI Web of Science on 01-23-2017.

The simple strength statistics can only give a preliminary impression of the study subject of experimental economics, and the relationship between the subjects needs to be reflected through the network form of the body analysis. We choose the threshold of 3 keywords co-occurrence data to make a co-word network map. In order to highlight the core of the network structure, the scattered network around the main components is not shown in the figure. The size of the node is consistent with the frequency of use of the keyword, and the higher the frequency, the more the color is red. In addition, the thickness of the link is consistent with the co-occurrence frequency between the keywords.

The pro-centricity degree of the overall study subject network figure is only 0.099 , that is, the theme does not focus on one key word or one sense-group. In other words, the discipline has reached a higher level of differentiation. The relationship between the existing research hot spots is relatively close, however, they also have a certain internal differentiation. For example, the three phrase: auctions, willingness to pay, risk aversion are more independent, while other key words have closer links. This is because auctions involve in more independent theoretical basis, and in the follow-up development process, it includes other secondary research topics like price seal auction, portfolio auction, and asymmetric auction. It is expected that the contact of auctions and its surrounding nodes will be more closely related to the development of other disciplines in the future, and the differentiation of other high frequency keywords will be more obvious. 


\section{Conclusion}

\section{Experimental Economics has Made Great Progress, but the Cluster Trend is not Significant.}

So far, experimental economics has entered the fourth stage of discipline development. It has been recognized by the academic mainstream and also has become a universal economic research method. Furthermore, the co-word network is relatively fragmented. There is no close convergence among the core concepts, but we also see that the network does not form a "cluster-like structure" based on different themes. This shows that experimental economics is rapidly growing after the initial stage, and the differentiation has not yet reached full.

The Simulation is Micro-Behavior-Based, and Macroeconomic Changes in the Simulation Capacity is Limited

Experimental economics representative branches: auctions, consumer behavior, and public goods supply are all about individual decisions, while experimental methods are good at recreating such behavior and making analysis and forecasting. However, for the complex variables, based on the impact of multiple individuals in different dimensions, the explanatory power of the experimental model is insufficient.

\section{Start from the Same Sense-Group of Research Changes, to the Jump Relationships among Relevant Sense-Groups}

The scope of the study is often limited by the recurrence of the same sense-group, and in-depth research sometimes hinders the exploration of the association type. With the help of the co-word network, the researchers' horizons are expanded and highly correlated sense-groups will be included in the study. This expansion can greatly enrich the integrity of the study.

\section{References}

[1] Cohen I B. Revolution in science[M]. Harvard University Press, 1985.

[2] Arthur W B. Self-reinforcing mechanisms in economics[J]. The economy as an evolving complex system, 1988, 5: 9-31.

[3] KUHN T S 2012. The structure of scientific revolutions [M]. University of Chicago press.

[4]Friedman D, Sunder S. Experimental methods: A primer for economists[M]. Cambridge University Press, 1994.

[5] PLOTT C R 1991. Will Economics Become an Experimental Science? Southern Economic Journal [J], 57: 901-919.

[6] CAMERER C F 1991. Does strategy research need game theory? Strategic management journal [J], 12: 137-152.

[7] RIZVI S A T 2005. Experimentation, general equilibrium and games [M] //R. L. PHILIPPE FONTAINE The Experiment in the History of Economics. Routledge: 50.

[8] KAGEL J H, ROTH A E 1995. The handbook of experimental economics [M]. Princeton university press Princeton, NJ.

[9] HERTWIG R, ORTMANN A 2001. Experimental practices in economics: A methodological challenge for psychologists? Behavioral and Brain Sciences [J], 24: 383-403.

[10] SIEGEL S 1961. Decision Making and Learning under Varying Conditions of Reinforcement. Annals of the New York Academy of Sciences [J], 89: 766-783.

[11] MILLER R M 2001. Paving wall street: Experimental economics and the quest for the perfect market [M]. John Wiley \& Sons. 
[12] LESK M E 1969. Word-word associations in document retrieval systems. American Documentation [J], 20: 27-38.

[13] Wasserman S, Faust K. Social network analysis: Methods and applications[M]. Cambridge university press, 1994.

[14] Yaoqi L, Lishan Xie 2013. Application and prospect of social network analysis in organizational management research. Chinese Journal of Management [J], 146-154. (In Chinese) 